\title{
Tipificação Epidemiológica dos casos de Leishmaniose Visceral Humana no Brasil, no período de 2013 A 2017
}

\author{
Epidemiological typification of human Visceral Leishmaniasis cases in Brazil, from 2013 to \\ 2017
}

Tipificación epidemiológica de los casos de Leishmaniasis Visceral humana en Brasil, de 2013 a 2017

Camila Rodrigues da Cunha ${ }^{1}$, Anselmo da Silva Ramos Filho ${ }^{1}$, Thiago Bezerra Lopes ${ }^{1}$, Maria Helena Mendonça de Araújo', Tatiana do Socorro dos Santos Calandrini' ${ }^{1}$, Maribel Nazaré dos Santos Smith Neves, Amanda Alves Fecury ${ }^{1}$, Rubens Alex de Oliveira Menezes ${ }^{1 *}$.

\section{RESUMO}

Objetivo: Traçar o perfil epidemiológico da Leshimaniose Visceral no Brasil de 2013 a 2017. Métodos: Estudo epidemiológico, retrospectivo, descritivo e analítico, nas regiões brasileiras, utilizando as variáveis sexo, raça, idade, escolaridade e evolução, em dados secundários coletados no DATASUS, tabulados em planilhas do programa Microsoft Excel 2010, para confecção e análise dos gráficos e tabelas. Resultados: Total de 18.733 notificações, com menor registro em 2016 (3.455 - 18,44\%) e maior em 2017 (4.515 - 29,10\%). A região nordeste totalizou $10.635(56,77 \%)$, o sudeste $3.660(19,53 \%)$, o norte $2.989(15,95 \%)$, o centro-oeste 1.396 $(7,45 \%)$ e o Sul com $53(0,28 \%)$ casos. Quanto ao gênero, $12.191(65,07 \%)$ eram homens e $6.541(34,91 \%)$ mulheres, pardos (13.291-70,94\%), predominando as idades entre 0 e 19 anos com 9.539 (50,92\%) casos em quatro das cinco regiões e, a maioria com ensino fundamental (5.176 -27,63\%), não tendo sido informado o grau de escolaridade em 11.703 (60,35\%). A evolução clínica mostrou cura em 12.639 (67,46\%) pacientes, óbito em $1.355(7,23 \%)$ e $2.923(15,60 \%)$ teve desfecho clínico ignorado ou em branco. Conclusão: Profissionais da saúde devem ser capacitados a intervir de forma preventiva, curativa e cuidadosa para que haja evolução favorável da doença, bem como notificação fidedigna dos casos.

Palavras-chave: Leishmaniose visceral, Epidemiologia, Brasil.

\begin{abstract}
Objective: To draw the epidemiological profile of visceral leshimaniasis in Brazil, from 2013 to 2017. Methods: Epidemiological, retrospective, descriptive and analytical study in the Brazilian regions, using the variables gender, race, age, education and evolution, in secondary data collected in the study. DATASUS, tabulated in Microsoft Excel 2010 spreadsheets, for analysis and preparation of graphs and tables. Results: Total 18,733 notifications, with the lowest record in 2016 (3,455 - 18.44\%) and the largest in 2017 (4,515 - 29,10\%). The northeast region totaled $10,635(56.77 \%)$, the southeast $3,660(19.53 \%)$, the north $2,989(15.95 \%)$, the midwest $1,396(7.45 \%)$ and the south with $53(0,28 \%)$ cases. Regarding gender, $12,191(65.07 \%)$ were men and 6,541 (34.91\%) women, brown (13,291-70.94\%), with ages between 0 and 19 years predominating with $9,539(50.92 \%)$ cases. in four of the five regions and most with elementary education $(5,176-27,63 \%)$, with $11,703(60,35 \%)$ having not been informed of the level of education. Clinical evolution showed cure in 12,639 $(67.46 \%)$ patients, death in 1,355 (7.23\%) and 2,923 (15.60\%) had an unknown or blank clinical outcome.
\end{abstract}

${ }^{1}$ Universidade Federal do Amapá (UNIFAP), Macapá - AP. *E-mail: rubens.alex@unifap.br 
Conclusion: Health professionals should be trained to intervene in a preventive, curative and careful manner so that there is favorable evolution of the disease, as well as reliable reporting of case

Keywords: Visceral leishmaniasis, Epidemiology, Brazil.

\section{RESUMEN}

Objetivo: Dibujar el perfil epidemiológico de la leshimaniasis visceral en Brasil, de 2013 a 2017. Métodos: Estudio epidemiológico, retrospectivo, descriptivo y analítico en las regiones brasileñas, utilizando las variables género, raza, edad, educación y evolución, en datos secundarios recogidos en el estudio. DATASUS, tabulado en hojas de cálculo de Microsoft Excel 2010, para análisis y preparación de gráficos y tablas. Resultados: 18,733 notificaciones totales, con el registro más bajo en 2016 (3,455 - 18.44\%) y el más grande en 2017 (4,515 - 29,10\%). La región noreste totalizó 10,635 (56.77\%), el sureste 3,660 (19.53\%), el norte $2,989(15.95 \%)$, el medio oeste $1,396(7.45 \%)$ y el sur con $53(0,28 \%)$ casos. En cuanto al género, 12,191 (65.07\%) eran hombres y 6,541 (34.91\%) mujeres, marrones (13,291-70.94\%), con edades entre 0 y 19 años predominando con 9,539 (50.92\%) casos. en cuatro de las cinco regiones y la mayoría con educación primaria (5,176-27,63\%), con nivel de educación no informado en 11,703 $(60,35 \%)$. La evolución clínica mostró cura en $12.639(67,46 \%)$ pacientes, muerte en 1.355 (7,23\%) y $2.923(15,60 \%)$ con resultado clínico desconocido o en blanco. Conclusión: Los profesionales de la salud deben estar capacitados para intervenir de manera preventiva, curativa y cuidadosa para que haya una evolución favorable de la enfermedad, así como la notificación confiable de casos.

Palabras clave: Leishmaniasis visceral, Epidemiología, Brasil.

\section{INTRODUÇÃO}

A Leishmaniose Visceral (LV) é uma doença crônica, ocasionada por protozoários da família Trypanosomatidae, do gênero Leishmania, e sua transmissão ocorre por meio da picada de um vetor flebotomíneo. No Brasil, o agente etiológico é a Leishmania (Leishmania) infantum, que faz parte do complexo Leishmania (Leishmania) donovani, e este possui um periodo de incubação de 10 dias a 24 meses (média de 2 a 6 meses) (BRASIL MS, 2014; SENA IVO, 2015).

Conjectura-se uma incidência mundial de aproximadamente 500.000 casos, 59.000 óbitos e 200 milhões de indivíduos sob o risco de adoecer, anualmente. Distribuída em 89 países, com atenção especial para as 06 principais regiões endêmicas: Brasil, Etiópia, Índia, Somália, Sudão do Sul e Sudão, que no ano de 2014 concentravam cerca de $90 \%$ dos casos no mundo (WHO, 2018; SILVA PL, et al., 2017).

No Brasil, o primeiro registro de LV foi em 1913, quando Migone, no Paraguai, esboçou o caso em material de necropsia de paciente proveniente de Boa Esperança, Mato Grosso, Brasil (SENA IVO, 2015). Até a década de 1950, foram feitas 379 notificações em 13 estados e, havia a crença de que a transmissão ocorria apenas em áreas rurais ou silvestres. No decorrer do período, correu o primeiro surto no país, em Sobral-CE. Nos anos subsequentes foram registrados na região, cerca de 1.000 novos casos. Entre 2000 a 2007 foram notificados 27.112 casos da doença no Sistema de Informação de Agravos de Notificações (SINAN) (BRASIL MS, 2014; PIRES B, et al., 2016).

Com intuito de reduzir o risco de transmissão, a taxa de letalidade e o grau de morbidade da LV nas áreas rurais e urbanas, foi instituído no Brasil, na década de 1950, o Programa de Vigilância e Controle da Leishmaniose Visceral (PVC-LV), que se fundamenta em três pilares: tratamento dos casos humanos, controle do reservatório canino e controle vetorial (MORAIS MHF, et al., 2015; SILVA T, 2017). Na última década, a vigilância epidemiológica evidencia como o fenômeno da urbanização e da periurbanização da LV influencia na distribuição da doença, antes tida como uma doença rural. Ressaltam-se os surtos ocorridos em áreas urbanas como no Rio de Janeiro (RJ), Belo Horizonte (MG) e Araçatuba (SP), e mais recentemente as epidemias ocorridas nas cidades de Três Lagoas (MS), Campo Grande (MS) e Palmas (TO) (BRASIL MS, 2014). 
Atualmente a LV está presente em todas as regiões, atingindo sobretudo indivíduos mais pobres e com dificuldade de acesso aos serviços de saúde, em particular a região Nordeste que é a mais carente e dispõe de condições ambientais propícias à proliferação dos vetores, representando mais de $90 \%$ dos casos notificados no país, sem distinção de vulnerabilidade entre gênero, etnia e idade, entretanto, crianças e idosos são mais propensos a adoecer (BRASIL MS, 2014; PAHO, 2018; SILVA PL, et al., 2017; BATISTA FMA, et al., 2014; OPAS, 2016).

A doença se caracteriza por um amplo espectro clínico, desde formas assintomáticas, oligossintomáticas até manifestações agudas clássicas, como febre prolongada, esplenomegalia, hepatomegalia, anorexia, edemas, anemia e perda ponderal. Na forma ligossintomática os sintomas são inespecíficos: febre baixa, diarreia, tosse seca, sudorese e adinamia persistente, associada a leve visceromegalia. Normalmente o baço é impalpável e o fígado está pouco aumentado. Na forma clássica, a febre se torna mais persistente a algumas vezes intermitente, com 2 a 3 picos diariamente; os distúrbios gastrointestinais (diarreia, disenteria ou obstipação) podem estar associados, além de adinamia, prostração, sonolência, mal-estar e progressiva perda ponderal. A tosse é seca ou pouco produtiva (DUARTE MIS e BADARÓ RS, 2015).

No agravamento da doença, sinais e sintomas como cabelos quebradiços, clássico sinal da bandeira (cabelos com duas ou três colorações), cílios alongados, caminhar vagaroso, edema de extremidades, hepatoesplenomegalia com aspecto de útero gravídico nas mulheres e cirrótico nos homens e esplenomegalia com o baço de consistência elástica ou algo endurecido, atingindo a cicatriz umbilical são achados frequentes (DUARTE MIS e BADARÓ RS, 2015).

O tratamento da LV inclui a terapêutica específica, realizada com antimoniais pentavalentes (ex.: antimoniato de meglutamina), que é a primeira escolha de tratamento, havendo exceção quando se trata de gestantes e outros pacientes que tenham contraindicação ao uso do antimoniato. Nesses casos, a única alternativa de tratamento é a anfotericina $B$, de preferência em sua formulação lipossomal. Ademais, são necessárias medidas de suporte adicionais (hidratação, antipiréticos, antimicrobianos, hemoterapia, suporte nutricional). Exames laboratoriais e eletrocardiogramas devem ser feitos durante o tratamento (BRASIL MS, 2017).

Os principais fatores associados ao óbito são alterações hematológicas (anemia, leucopenia e plaquetopenia), junto às infecções bacterianas (do trato urinário, respiratório, piodermites e otite média aguda), que se não forem medicadas de forma rápida e pertinente, o paciente pode desenvolver um quadro séptico com evolução letal. Pacientes na fase clássica com complicações que agravem a doença também podem evoluir para óbito. Casos de infecções oportunistas em portadores da Síndrome de Imunodeficiência Adquirida (SIDA/AIDS) tem ocorrido. Em geral, a evolução da doença apresenta um bom prognóstico em pacientes previamente sadios, principalmente quando diagnosticada e tratada precocemente. O mesmo não é observado em pacientes imunocomprometidos (AGUIAR PF e RODRIGUES RK, 2017; SILVA PL, et al., 2017; BRASIL MS, 2017).

A vigilância epidemiológica objetiva ratificar e apurar os casos, diminuir as taxas de letalidade e grau de morbidade através do diagnóstico e tratamento precoce, bem como reduzir os riscos de transmissão por meio do monitoramento da população de reservatórios e do agente transmissor, pois a LV é uma doença de notificação compulsória que deve ser registrada no Sistema de Informação de Agravos de Notificações (SINAN) logo que for diagnosticada (SILVA PL, et al., 2017).

Adicionalmente, observa-se que a doença tem apresentado uma ampliação para áreas urbanas de médios e grandes portes, levando a uma grande expansão geográfica e problema de saúde pública. Além disso, é importante considerer aspectos responsáveis pela dispersão da doença como: fatores relacionados ao uso do solo, agricultura, tipo de vegetação e desmatamento, planejamento das cidades e de ocupações humanas, incluindo o movimento e composição populacional humana, tal qual as condições de vida, a infraestrutura sanitária e o modo de vida dos indivíduos (FARIAS FTG at al., 2019).

Nesse sentido, este estudo tem como objetivo traçar o perfil epidemiológico da leishmaniose visceral humana no Brasil, de 2013 a 2017, expondo as principais nuances de sua distribuição nas regiões brasileiras 
como sexo, raça, idade, escolaridade, assim como sua evolução, morbidade e letalidade, visando orientar os profissionais da saúde para uma melhor vigilância e controle.

\section{MÉTODOS}

Trata-se de um estudo epidemiológico, retrospectivo, descritivo e analítico de uma série histórica nas regiões brasileiras, no período de 2013 a 2017. Foram utilizados dados secundários obtidos no site do Departamento de Informática do Sistema Único de Saúde (DATASUS) (www2.datasus.gov.br), selecionado "Informações de Saúde do Tabulador de Dados do Sistema Único de Saúde (TABNET), Epidemiologia e Morbidade" e doravante a opção "Doenças e Agravos de Notificação" - De 2007 em diante no Sistema de Informação de Agravos de Notificação (SINAN), escolhido o item Leishmaniose Visceral e, então, foram compiladas as informações das regiões do Brasil de 2013 a 2017, com as variáveis sexo, raça, idade, escolaridade e evolução.

Os dados supracitados foram tabulados em planilhas do programa Microsoft Excel 2017 (Versão Windows 10) para análise e confecção dos gráficos e tabelas. A pesquisa trata, apenas, de dados secundários, de domínio público e de livre acesso, dispensando a submissão e aprovação por comitê de ética em Pesquisa. Mas, ressalta-se que foram tomados os cuidados éticos que preceituam a Resolução 466/12, do Conselho Nacional de Saúde.

\section{RESULTADOS E DISCUSSÃO}

No Brasil, o total de casos de Leishmaniose Visceral Humana (LVH) notificados de 2013 a 2017 foi de 18.733, perfazendo uma média de 3.746,6 por ano. O ano de 2016 foi o que apresentou menor número de casos, e o de 2017 o maior número, com respectivamente, 3.455 (18,44\%) e 4.515 (24,10\%) notificações que se encontram no (Gráfico 1). Esse dado concorda com o Guia de Vigilância em Saúde (2017), que avaliou a situação epidemiológica no período de 2003 a 2012, onde a média anual foi de 3.565 casos. Vale ressaltar que esses valores podem variar em casos de epidemias esporádicas (BRASIL MS, 2017).

Gráfico 1 - Distribuição dos casos de Leishmaniose Visceral Humana no período de 2013 a 2017 no Brasil.

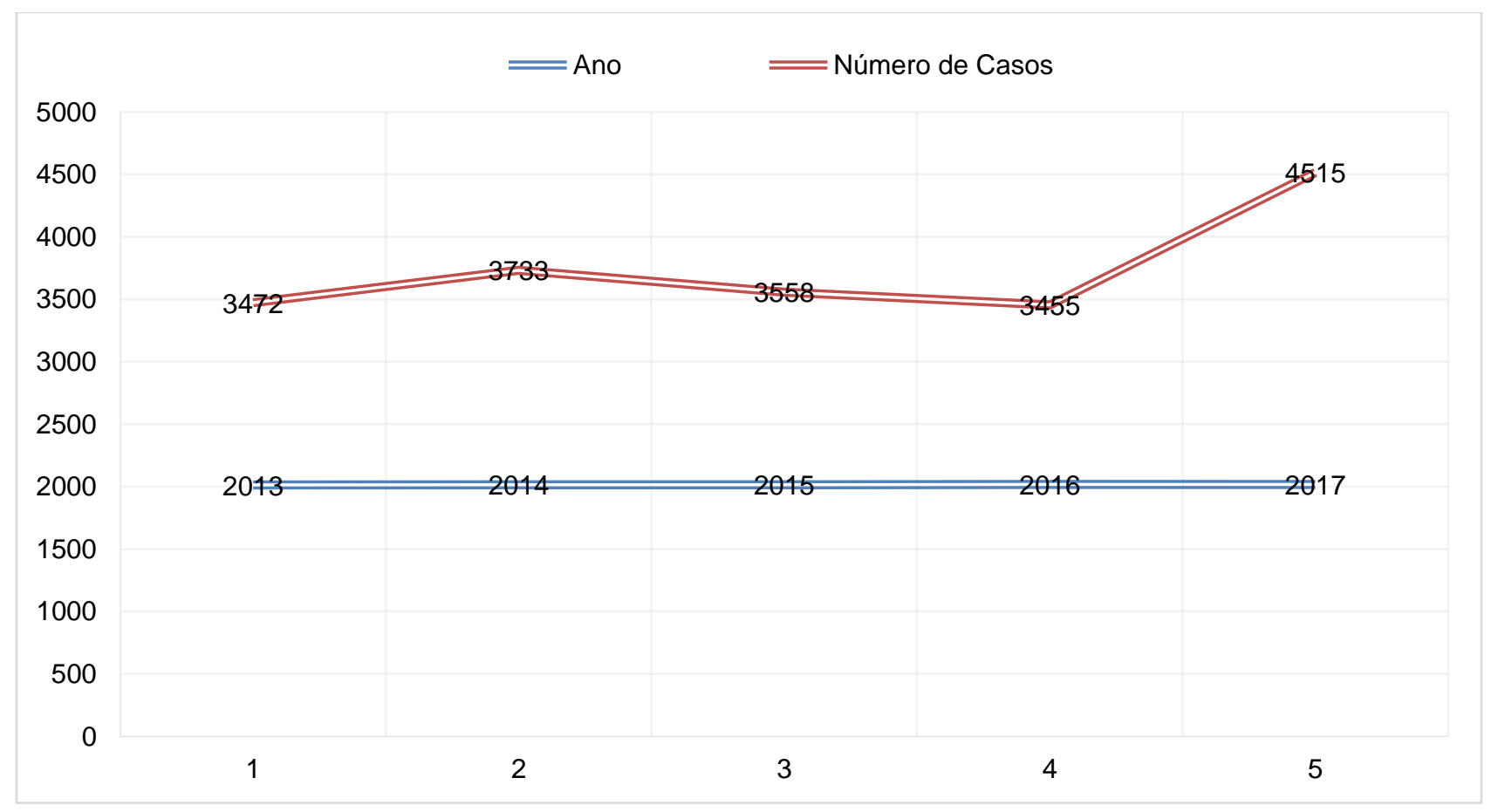

Fonte: Cunha CR, et al., 2019. 
Ao analisar o número de casos por região, o Nordeste notificou 10.635 (56,77\%) casos, apresentando uma incidência média de 3,75 casos a cada 100 mil habitantes, no período estudado; Sudeste com 3660 (19,53\%), incidência média de 0,84 casos a cada 100 mil habitantes; Norte com 2.898 (15,95\%), incidência média de 3,40 casos a cada 100 habitantes; Centro-Oeste $1.396(7,45 \%)$ casos com incidência média de 1,80 casos a cada 100 mil habitantes; e Sul com $53(0,28 \%)$, incidência média de 0,034 casos a cada 100 mil habitantes, demonstrados (Gráfico 2).

Os fatores relacionados ao aparecimento da doença nas regiões metropolitanas gaúcha foram citados por Teixeira MS (2014) que realizou um estudo na cidade de Porto Alegre e observou que houve uma expansão desordenada com aparecimento de bairros com grande número de casas e pequenos sítios com áreas extensas de mata verde entre residências, semelhante ao observado em áreas rurais, com vulnerabilidade social, presença de lixo mal acondicionado, falta de saneamento básico nas residências e presenças de cães errantes. As mudanças climáticas que tem ocorrido ao longo dos anos na região sul, com estações do ano já não tão definidas foram mencionados por pesquisadores na reportagem de British Broadcasting Corporation (BBC) Brasil (TEIXEIRA MC, 2014; SEIBT, 2017).

Ao se comparar com estudos prévios, nas décadas de 80 e 90, a região Nordeste agrupava cerca de $90 \%$ dos casos de Leishmaniose Visceral, segundo Barbosa (2013), contudo, pode-se observar uma expansão da doença no território nacional, principalmente para as regiões Norte, Sudeste e Centro-oeste, de acordo com o artigo de Von Zuben e Donalísio (2016).

Sendo assim, é possível atribuir fatores associados ao processo migratório, esvaziamento rural, altas densidades populacionais, ocupação desordenada de regiões periféricas, agressões ao meio ambiente, pressões econômicas ou sociais à essa maior distribuição da doença pelo país, com a expansão das áreas endêmicas favorecendo o aparecimento de novos focos. (BARBOSA IR, 2013; VON ZUBEN APB e DONALÍSIO MR, 2016; SOUSA NA, et al., 2018).

Gráfico 2 - Distribuição dos casos de Leishmaniose Visceral Humana, notificados nas regiões do Brasil, período de 2013 a 2017.

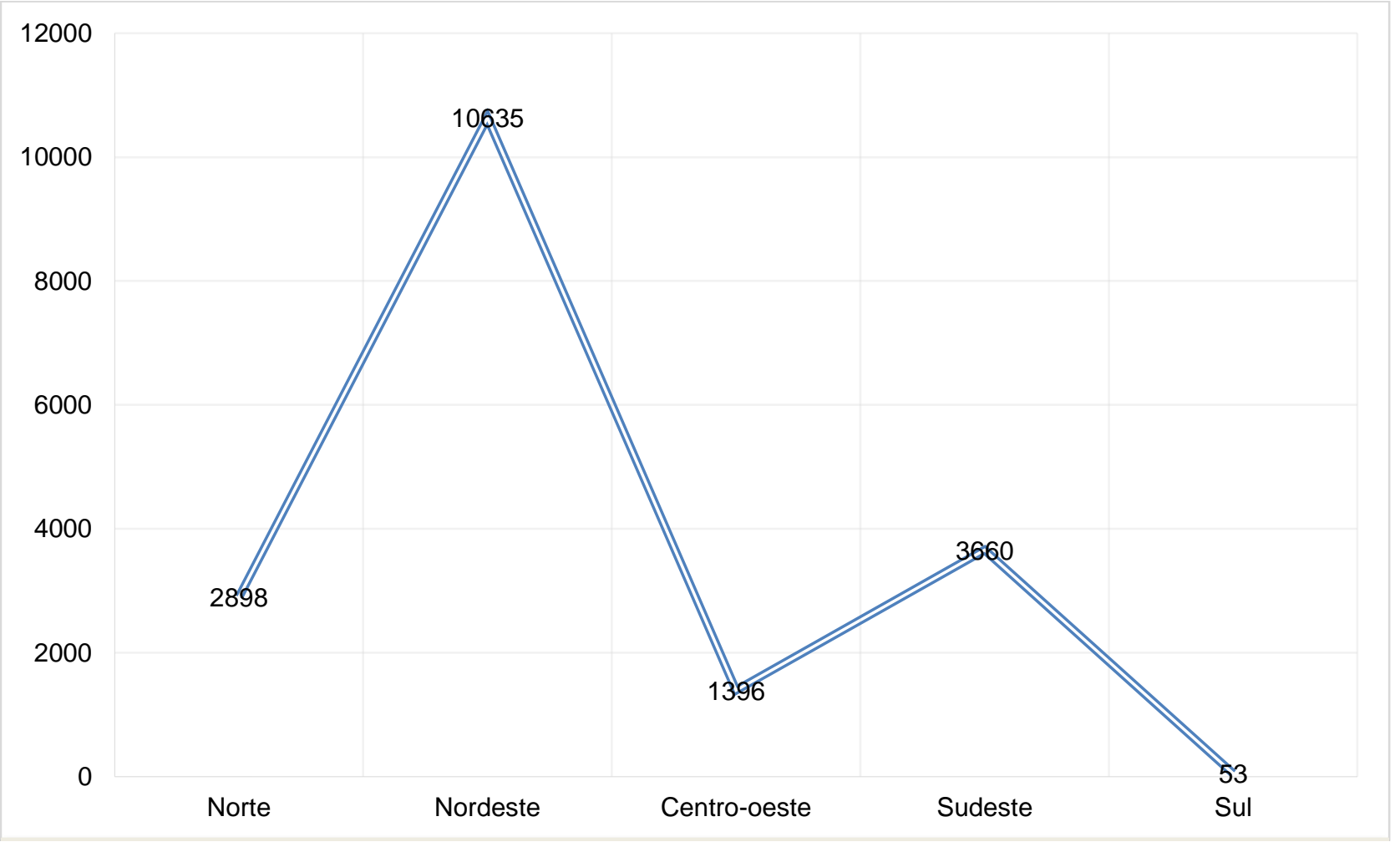

Fonte: Cunha CR, et al., 2019. 
Observa-se na Tabela 1, que na série histórica de 2013 a 2015, a região Nordeste apresentava a maior incidência de casos de LVH, seguida, do Norte. Entretanto, houve mudança de padrão nos anos 2016 e 2017, onde a maior incidência passou a ser na região Norte do país. Apesar do maior percentual da doença ocorrer na região Nordeste, ao se analisar as taxas demográficas de cada região, através do censo IBGE (2010), nota-se mudança no padrão de disseminação da doença.

Entre 2013 e 2015, as maiores taxas de incidência decorriam da região Nordeste (3,55/4,31/3,79). Já nos anos de 2016 e 2017 a incidência mais exuberante foi oriunda da região Norte $(3,51 / 4,76)$. Ao considerar a incidência média no período estudado, a região Nordeste apresenta maior montante, com 3,75 casos a cada 100 mil habitantes, e a região Sul o menor, com 0,034 casos a cada 100 mil habitantes (Tabela 1). A incidência média no Brasil no mesmo período foi de 1,8 casos a cada 100 mil habitantes. Não foram encontrados estudos prévios para fins de comparação (IBGE, 2010).

Tabela 1 - Incidência anual de Leishmaniose Visceral Humana a cada 100 mil habitantes, notificados no Brasil, de 2013 a 2017.

\begin{tabular}{lcccccc}
\hline Região/Ano & $\mathbf{2 0 1 3}$ & $\mathbf{2 0 1 4}$ & $\mathbf{2 0 1 5}$ & $\mathbf{2 0 1 6}$ & $\mathbf{2 0 1 7}$ & Média \\
\hline Norte & 3,36 & 2,51 & 2,89 & 3,51 & 4,76 & 3,4 \\
Nordeste & 3,55 & 4,31 & 3,79 & 3,21 & 3,93 & 3,75 \\
Centro-oeste & 2,38 & 1,83 & 1,51 & 1,48 & 1,84 & 1,8 \\
Sudeste & 0,65 & 0,69 & 0,77 & 0,87 & 1,25 & 0,84 \\
Sul & 0,013 & 0,02 & 0,02 & 0,057 & 0,06 & 0,034 \\
Brasil & 1,72 & 1,84 & 1,74 & 1,67 & 2,17 & 1,82 \\
\hline
\end{tabular}

Fonte: Cunha CR, et al., 2019.

Quanto ao gênero, no Brasil, 12.191 (65,07\%) eram homens, 6.541 (34,91\%) mulheres, e 1 (0,0053\%) teve o gênero ignorado em sua notificação. A razão média entre os gêneros masculino e feminino nas regiões brasileiras foi de 13:7, aproximadamente. O masculino foi predominante em todas as regiões, conforme mostra a (Tabela 2), confirmando o que já foi evidenciado em estudos como o de Rangel O, et al. (2015), Barbosa IR (2013) e Góes MAO (2012) mostram que essa maior prevalência está relacionada com fatores hormonais e exposição ao vetor (RANGEL O, et al., 2015; BARBOSA IR, 2013; GOES MAO, et al., 2012).

Adicionalmente, esse contexto evidencia que os indivíduos do gênero masculino estão mais propícios a LV em decorrência da desatenção em relação aos fatores de riscos, aos meios preventivos e não procura das unidades de saúde, divergente das mulheres que buscam mais os serviços das unidades de saúde (SANTOS ESM, et al., 2019). Quanto à faixa etária, 9.539 (50,92\%) tinham de 0 a 19 anos, 4.306 (22,98\%) estavam entre 20 e 39 anos, 3.344 (17,85\%) estavam entre 40 e 59 anos de idade, e 1.538 (8,21\%) tinham 60 anos ou mais. Nas regiões Norte, Nordeste, Sudeste e no Centro-oeste houve predominância na faixa etária 0 a 19 anos de idade; na região Sul, a mais predominante foi de 20 a 39 anos, apresentados na (Tabela 2).

Neste estudo houve predomínio da idade de 0 a 19 anos (50,92\%), o que pactua com a pesquisa Rangel O, et al. (2015) e com a de Góes MAO (2012). Vale ressaltar que ocorre um estreitamento na base da pirâmide etária brasileira, onde se situa esse grupo, em virtude da diminuição da taxa de natalidade em curso. Esse fato é demonstrado no censo do Instituto Brasileiro de Geografia e Estatistica (IBGE) (2010), onde essa faixa etária englobava $33,1 \%$ da população brasileira, ao passo que a faixa etária compreendida entre 20 e 39 anos de idade já representava $33,3 \%$ da população do país.

O Guia de Vigilância em Saúde (2017) chama atenção para os indivíduos menores de 10 anos, e cita que a razão da maior suscetibilidade em crianças é explicada pelo estado de relativa imaturidade imunológica celular, agravado pela desnutrição, tão comum nas áreas endêmicas, além de uma maior exposição ao vetor no peridomicílio (BRASIL MS, 2017; GÓES MAO, et al., 2012; IBGE, 2010; RANGEL O, et al., 2015). 
Tabela 2 - Distribuição dos casos de Leishmaniose Visceral Humana de acordo com gênero e idades nas regiões do Brasil, no período de 2013 a 2017.

\begin{tabular}{|c|c|c|c|c|}
\hline \multirow[b]{3}{*}{ Região } & \multicolumn{4}{|c|}{ SEXO } \\
\hline & \multicolumn{2}{|c|}{ Masculino } & \multicolumn{2}{|c|}{ Feminino } \\
\hline & (n) & $(\%)$ & (n) & $(\%)$ \\
\hline Norte & 1828 & 61,14 & 1161 & 38,86 \\
\hline Nordeste & 7036 & 66,15 & 3599 & 33,85 \\
\hline Centro-oeste & 908 & 65,04 & 487 & 34,96 \\
\hline Sudeste & 2384 & 65,133 & 1276 & 34,87 \\
\hline Sul & 35 & 66,03 & 18 & 33,97 \\
\hline \multirow[t]{3}{*}{ Brasil } & 12.191 & 65,07 & 6541 & 34,91 \\
\hline & \multicolumn{4}{|c|}{ FAIXA ETÁRIA } \\
\hline & 0 a 19 anos & 20 a 39 anos & 40 a 59 anos & $>60$ anos \\
\hline Região & $\%(n)$ & $\%(\mathbf{n})$ & $\%(\mathbf{n})$ & $\%(n)$ \\
\hline Norte & $61,93(1856)$ & $21,05(631)$ & $12,18(356)$ & $4,84(145)$ \\
\hline Nordeste & $53,74(5713)$ & $23,14(2460)$ & $16,4(1744)$ & $6,72(714)$ \\
\hline Centro-oeste & $36,56(510)$ & $25,88(361)$ & 23,94 (334) & $13,62(190)$ \\
\hline Sudeste & $39,48(1445)$ & 22,9 (838) & $24,62(901)$ & $13(476)$ \\
\hline Sul & $28,3(15)$ & $30,19(16)$ & $16,98(9)$ & $24,53(13)$ \\
\hline Brasil & 50,91 (9539) & $22,98(4306)$ & $17,9(3353)$ & $8,21(1538)$ \\
\hline
\end{tabular}

Fonte: Cunha CR, et al., 2019.

Quanto à distribuição por grupos étnicos, houve predomínio da população parda, com 13.291 (70,94\%), seguida pela branca com 2.324 (12,40\%), preta com $1.567(8,36 \%)$, indígena, com $243(1,29 \%)$ e amarela, com $124(0,66 \%)$ casos. Foram ignorados $1.184(6,32 \%)$ dos casos, apresentados na (Tabela 3). Acerca dos grupos étnicos, escolaridade e desfecho clínico, não foram encontrados estudos a fim de se realizar comparativos epidemiológicos. O que se pode evidenciar é um maior predomínio da população parda (70, $94 \%$ ) em 4 das 5 regiões brasileiras. Esse dado pode ser atribuído à forte miscigenação que ocorreu em nosso país ao longo da história.

Porém, de acordo com o censo demográfico IBGE (2010), ao se analisar a composição étnica da população brasileira, levando-se em consideração a autodeclaração, foram obtidos os seguintes resultados: $47,7 \%$ branca, $46,5 \%$ parda, $7,6 \%$ preta, $1,09 \%$ amarela e $0,42 \%$ se indígena. Por se tratar de autodeclaração isto constitui um problema por não inferir adequadamente a realidade da população, daí ocorre uma discordância entre os dados (IBGE, 2010).

$\mathrm{Na}$ escolaridade, o total de notificações preenchidas corretamente foi de 7.426. Observou-se que 547 $(7,72 \%)$ dos indivíduos foram classificados como analfabetos, $5.176(51,76 \%)$ tinham ensino fundamental, $1.487(20,02 \%)$ ensino médio, e 189 (2,54\%) ensino superior. Observa-se que $11.307(60,35 \%)$ tiveram a escolaridade ignorada. Excluindo-se esses casos em que a escolaridade foi ignorada ou não se aplicava, em todas as regiões brasileiras houve predomínio do ensino fundamental, seguido pelo ensino médio e, por último, ensino superior. A distribuição dos casos por escolaridade nas regiões brasileiras pode ser observada na (Tabela 3).

Sobre os índices de instrução, há preponderância dos níveis de escolaridade mais básicos, como número de pacientes com ensino fundamental completo que representam $27,63 \%$ do total de casos. Sobre este dado, pode-se especular uma correlação entre baixos níveis socioeconômicos, baixa escolaridade e, por consequência, menor acesso à informação (ex.: educação em saúde) e saneamento básico. 
Tabela 3 - Distribuição dos casos de Leshmaniose Visceral Humana notificados por raça e escolaridade nas regiões do Brasil, período de 2013 a 2017.

\begin{tabular}{lccccc}
\hline & \multicolumn{5}{c}{ RAÇA } \\
\cline { 2 - 6 } Região & Branca & Preta & Amarela & Parda & Indigena \\
\cline { 2 - 6 } Norte & $\%(\mathbf{n})$ & $\%(\mathbf{n})$ & $\%(\mathbf{n})$ & $\%(\mathbf{n})$ & $\%(\mathbf{n})$ \\
Nordeste & $8,58(249)$ & $7,27(211)$ & $0,62(18)$ & $80,88(2347)$ & $2,65(77)$ \\
Centro-oeste & $8,26(838)$ & $8,06(818)$ & $0,65(66)$ & $81,79(8298)$ & $1,24(126)$ \\
Sudeste & $25,18(311)$ & $8,91(110)$ & $0,81(10)$ & $63,56(785)$ & $1,54(19)$ \\
Sul & $27,96(899)$ & $13,03(419)$ & $0.9(29)$ & $57,45(1847)$ & $0,65(21)$ \\
Brasil & $52,94(27)$ & $17,65(9)$ & $1,96(1)$ & $27,45(14)$ & $0(0)$ \\
\hline & $13,24(2324)$ & $8,93(1567)$ & $0,71(124)$ & $75,74(13291)$ & $1,38(243)$ \\
Região & Analfabeto & Fundamental & Médio & \\
\hline Norte & $\%, 61(77)$ & $69,18(806)$ & $21,2(247)$ & \\
Nordeste & $9,27(392)$ & $70,65(2985)$ & $18,55(784)$ & $1,51(64)$ & \\
Centro-oeste & $4,16(26)$ & $62,66(391)$ & $26,12(163)$ & $7,05(44)$ & \\
Sudeste & $5,62(78)$ & $70,8(982)$ & $20,4(283)$ & $3,17(44)$ & \\
Sul & $4(1)$ & $48(12)$ & $40(10)$ & $8(2)$ & \\
Brasil & $7,72(547)$ & $69,7(5176)$ & $20,02(1487)$ & $2,54(189)$ & \\
\hline
\end{tabular}

Fonte: Cunha CR, et al., 2019.

Considerando o desfecho clínico, nota-se que somente $67,46 \%$ dos pacientes evoluíram para a cura, demonstrando uma redução nos números, visto que, no período 2008 - 2012 havia uma taxa de cura de $72,2 \%$, segundo dados do SINAN (DATASUS, 2018).

Para a escolaridade e desfecho clínico, foi encontrado elevado quantitativo de não preenchimento destes (ignorado ou deixado em branco), fator que desperta a discussão sobre a importância de as notificações serem preenchidas de forma completa.

Observa-se uma menor taxa na região Norte $(4,60 \%)$ e a maior taxa na região Sul (14,39\%). Esse número mais expressivo na região Sul pode não ocorrer por uma razão específica, mas pode-se especular que seja porque a incidência da LVH na população é tão diminuta, que qualquer óbito registrado passa a ter mais relevância.

Uma segunda hipótese também levaria em consideração a pequena incidência nessa localidade, visto que, alguns casos podem passar absortos quando se considera o grande leque de diagnósticos diferenciais. Esta suposição não significa que a assistência/tratamento na região Sul seja mais deficiente, mas talvez os casos clínicos resolvidos sejam mais subnotificados para Leishmaniose Visceral Humana que nas demais regiões.

Em relação a evolução, 12.639 (67,44\%) pacientes ficaram curados, 1.355 (7,23\%) foram a óbito, 2.923 $(15,58 \%)$ tiveram seu desfecho clínico ignorado ou deixado em branco, $138(0,72 \%)$ pacientes abandonaram o tratamento, enquanto que os óbitos por outras causas e transferência totalizaram $1.678(8,95 \%)$ dos casos notificados. A distribuição da evolução está descrita na (Tabela 4).

Analisando-se a letalidade no período estudado, foi encontrada uma média de 7,24\% no Brasil. Por região, encontra-se o Norte com 4,60\% dos casos; Nordeste com 7,10\%; Centro-oeste com 7,69\%; Sudeste com 9,45\%; e Sul com 14,39\%. A descrição da letalidade em porcentagem pode ser observada na Tabela 4. 
Tabela 4 - Distribuição dos casos de Leishmaniose Visceral por evolução e letalidade nas regiões do Brasil, notificados de 2013 a 2017.

\begin{tabular}{|c|c|c|c|c|c|c|}
\hline \multirow[b]{3}{*}{ Região } & \multicolumn{6}{|c|}{ EVOLUÇÃO } \\
\hline & \multirow{2}{*}{$\begin{array}{l}\text { Cura } \\
\%(n)\end{array}$} & \multirow{2}{*}{$\begin{array}{c}\text { Óbito por } \\
\text { LV } \\
\%(n)\end{array}$} & \multirow{2}{*}{$\begin{array}{c}\text { Ignorado } \\
\%(n)\end{array}$} & \multirow{2}{*}{$\begin{array}{c}\text { Abandono } \\
\%(n)\end{array}$} & \multicolumn{2}{|c|}{ Outros } \\
\hline & & & & & \multicolumn{2}{|c|}{$\%(n)$} \\
\hline Norte & $11,9(22231)$ & $0,72(135)$ & $2,16(405)$ & $0,16(30)$ & \multicolumn{2}{|c|}{$1(188)$} \\
\hline Nordeste & $34,37(6440)$ & $4,01(753)$ & $11,42(2140)$ & $0,42(79)$ & \multicolumn{2}{|c|}{$6,52(1223)$} \\
\hline Centro-oeste & $5,6(1050)$ & $0,57(108)$ & $0,62(117)$ & $0,06(12)$ & \multicolumn{2}{|c|}{$0,58(109)$} \\
\hline Sudeste & $15,42(2889)$ & $1,85(348)$ & $1,33(250)$ & $0,08(16)$ & \multicolumn{2}{|c|}{$0,83(157)$} \\
\hline Sul & $0,15(29)$ & $0,05(11)$ & $0,05(11)$ & $0(1)$ & \multicolumn{2}{|c|}{$0(1)$} \\
\hline Brasil & $67,44(12639)$ & $7,2(1355)$ & $15,58(2923)$ & $0,72(138)$ & \multicolumn{2}{|c|}{$8,93(1678)$} \\
\hline & \multicolumn{6}{|c|}{ LETALIDADE } \\
\hline Região & 2013 & 2014 & 2015 & 2016 & 2017 & Média \\
\hline Norte & $5,76 \%$ & $3,68 \%$ & $6,12 \%$ & $3,85 \%$ & $3,62 \%$ & 4,60 \\
\hline Nordeste & $5,74 \%$ & $6,35 \%$ & $7,72 \%$ & $8,36 \%$ & $7,36 \%$ & 7,10 \\
\hline Centro-oeste & $9,24 \%$ & $7,85 \%$ & $9,82 \%$ & $6,46 \%$ & $5,11 \%$ & 7,69 \\
\hline Sudeste & $9,36 \%$ & $9,30 \%$ & $9,03 \%$ & $9,78 \%$ & $9,78 \%$ & 9,45 \\
\hline Sul & 0 & $16,66 \%$ & 0 & $35,29 \%$ & $20 \%$ & 14,39 \\
\hline Brasil & $6,68 \%$ & $6,64 \%$ & $7,86 \%$ & $7,87 \%$ & $7,15 \%$ & 7,24 \\
\hline
\end{tabular}

Fonte: Cunha CR, et al., 2019.

Diante desse contexto epidemiológico, a efetividade das medidas de prevenção e controle depende do conhecimento dos indicadores epidemiológicos e operacionais, sendo adequadas a cada foco em particular, avaliando estratégias que permitam otimizar recursos e esforços nos programas de vigilância epidemiológica da LVH afim de reduzir os índices de morbidade e letalidade da doença (TRINDADE EL et al., 2019; SANTOS ESM, et al., 2019).

As estratégias de controle devem ser flexíveis e com foco para cada região, considerando a grande extensão territorial do pais e suas características como a variedade de agentes, reservatórios e de vetores, elucidando a complexidade do controle dessa enfermidade (SOUZA APF et al., 2018). O percalço no registro dos casos de LVH pode estar relacionado também a fatores como os sintomas serem semelhantes aos de outras doenças febris em áreas endêmicas e os recursos diagnósticos e de boa vigilância serem limitados nessas áreas.

\section{CONSIDERAÇÕES FINAIS}

A Leishmaniose Visceral está presente em áreas urbanas e rurais de todas as regiões brasileiras, com aumento do número de casos em regiões onde não ocorriam anteriormente, atingindo uma população vulnerável, devendo as equipes de saúde serem capacitadas a intervir de forma preventiva e curativa, para que haja evolução favoravel dos casos. Espera-se que este trabalho contribua para um maior conhecimento dos padrões atuais de acometimento da doença (observando parâmetros como idade, sexo, grupo étnico, escolaridade e desfecho clínico) para assim, traçar melhores estratégias no manejo da patologia e nas intervenções dos órgãos competentes. Sendo assim, aspira-se um aumento dos índices de cura e controle de disseminação da doença, bem como a notificação fidedigna dos casos. 


\section{REFERÊNCIAS}

1. AGUIAR PF, RODRIGUES RK. Leishmaniose visceral no Brasil: artigo de revisão. Revista Unimontes Científica. Montes Claros, 2017; 19(1): 191-204.

2. BATISTA FMA, et al. Leishmaniose: perfil epidemiológico dos casos notificados no estado do Piauí entre 2007 e 2011. Revista UNIVAP. São Paulo, 2014; 20(35): 44-55.

3. BRASIL. Ministério da Saúde. Secretaria de Vigilância em Saúde. Departamento de Vigilância Epidemiológica. Manual de vigilância e controle da leishmaniose visceral, ed. 1, reimp. 5. Brasília: Ministério da Saúde; 2014.

4. BRASIL. Ministério da Saúde, Secretaria de Vigilância em Saúde, Coordenação-Geral de Desenvolvimento da Epidemiologia em Serviços. Guia de Vigilância em Saúde, v. 3, ed. 1. Brasília: Ministério da Saúde; 2017.

5. BARBOSA IR. Epidemiologia da leishmaniose visceral no estado do Rio Grande do Norte, Brasil. Revista Epidemiologia e Controle de Infecção. Rio Grande do Norte, 2013; 3(1):17-21.

6. DATASUS. Informações de Saúde (TABNET). Epidemiológicas e Morbidade. Doenças e Agravos de Notificação. De 2007 em diante (SINAN): Leishmaniose Visceral.

7. DUARTE MIS; BADARÓ RS. Veronesi: Tratado de Infectologia. Ed. 5. São Paulo: Atheneu, 2015.

8. FARIAS FTG, at al. Perfil epidemiológico de pacientes diagnosticados com leishmaniose visceral humana no Brasil. C\&D-Revista Eletrônica da FAINOR, 2019; 12 (3): 485-501.

9. GÓES MAO, et al. Série temporal da leishmaniose visceral em Aracaju, estado de Sergipe, Brasil (1999 a 2008): aspectos humanos e caninos. Revista Brasileira de Epidemiologia. São Paulo, 2012; 15(2): 298-307.

10. IBGE. Instituto Brasileiro de Geografia e Estatística. Censo demográfico 2010: População estimada. Brasília.

11. MORAIS MHF, et al. Evaluation of visceral leishmaniasis control activities in Belo Horizonte, Minas Gerais, Brazil, 2006-2011. Revista Epidemiologia e Serviços de Saúde. Brasília, 2015; 24(3): 485-496.

12. OPAS. Organização Pan-Americana da Saúde: Leishmanioses: Informe Epidemiológico nas Américas. Washington: Organização Pan-Americana da Saúde; 2018.

13. PAHO. Pan American Health Organization: Leishmaniases: Epidemiological Report in the Americas: Washington: Pan American Health Organization; 2016.

14. PIRES BS, et al. Fatores Epidemiológicos da Leishmaniose Visceral Humana no Brasil, 2008-2015. Simpósio de TCC e Seminário de IC. 2016; 1955-1965.

15. RANGELL O, et al. Leishmaniose visceral no estado de São Paulo: Tendência geral da letalidade entre 1999 a 2013 e o risco de óbitos por estratificação epidemiológica dos municípios e regionais de Vigilância Epidemiológica entre 2011 a 2013. Boletim Epidemiológico Paulista. São Paulo, 2015; 12(143): 1-8.

16. SANTOS ESM, et al. Aspectos Epidemiológicos da Leishmaniose Visceral. Revista Eletrônica Acervo Saúde, 2019; Vol.Sup.23: (e959): 1-5.

17. SEIBT T. Por que a leishmaniose avança no sul do Brasil e o que os cães têm a ver com isso. BBC, News Brasil. Porto Alegre, 27 de outubro de 2017.

18. SENA IVO. Fatores associados ao óbito por leishmaniose visceral em hospital público de referência no estado do Piauí. Dissertação (Mestrado em Saúde Pública) - Escola Nacional de Saúde Pública Sergio Arouca, Rio de Janeiro, 2015; 64 p.

19. SILVA PL, et al. Epidemiologia da Leishmaniose Visceral em um município da Bahia. Revista Saúde.com, 2017; 13(3): 933-940.

20. SILVA T. Leishmaniose visceral: análise espaço-temporal, avaliação do perfil clínico-epidemiológico e fatores associados ao óbito em Belo Horizonte e Minas Gerais. Dissertação (Doutorado em Parasitologia) - Instituto de Ciências Biológicas. Universidade Federal de Minas Gerais, Minas Gerais, 2017; 172 p.

21. SOUZA APF, et al. Aspectos clínicos e epidemiológicos da leishmaniose tegumentar Americana. Revista Eletrônica Acervo Saúde, 2018. Vol. Sup. 3, S1535-S1541.

22. TEIXEIRA MC. Soroepidemiologia de Leismania (L.) chagasi em cães no município de Porto Alegre, Rio Grande do Sul. Tese (Doutorado) - Universidade Federal do Rio Grande do Sul, Faculdade de Veterinária, Programa de PósGraduação em Ciências Veterinárias, Porto Alegre, BR-RS, 71f. 2014.

23. TRINDADE EL et al. Desafios para o controle da leishmaniose visceral humana no Pará. Braz. J. Hea. Rev., 2019;2 (6): 5488-5499.

24. VON ZUBEN APB, DONALÍSIO MR. Dificuldades na execução das diretrizes do Programa de Vigilância e Controle da Leishmaniose Visceral em grandes municípios brasileiros. Cadernos de Saúde Pública. Rio de Janeiro, 2016; 32(6): e00087415.

25. WHO. Leishmaniasis: the disease and its epidemiology. Leishmaniasis Home.

26. WHO. Leishmaniasis: the disease and its epidemiology. Epidemiology. 